\title{
A Closer Scientific Look at Foam Bitumen
}

\author{
Biruk W. Hailesilassie ${ }^{1}$, Iwan Jerjen ${ }^{2}$, Michele Griffa ${ }^{3}$, Manfred N. Partl ${ }^{4}$ \\ ${ }^{1}$ Infrastructure Engineering, School of Architecture and the Built Environment, Royal Institute of \\ Technology (KTH), SE- 10044 Stockholm, Sweden, biruk.hailesilassie@abe.kth.se \\ ${ }^{2}$ Institute for Biomedical Engineering, ETHZ, Gloriastrasse 35, CH-8092 Zurich, Switzerland, \\ iwan.jerjen@psi.ch
}

${ }^{3}$ Concerete and Construction Chemistry Laboratory, center for X-ray analytics, EMPA, Swiss Federal Laboratories for Material Science and Technology,CH-8600 Duebendorf, Ueberlandstrasse 129, Switzerland, Michele.Griffa@empa.ch

${ }^{4}$ KTH Stockholm, School of Architecture and the Built Environment, Director Road Engineering/Sealing Components, EMPA, Swiss Federal Laboratories for Material Science and Technology,CH-8600 Duebendorf, Ueberlandstrasse 129, Switzerland, manfred.partl@empa.ch

\begin{abstract}
In the asphalt industry a substantial interest is observed to find possibilities to reduce the production temperature of asphalt mixtures. In the context of this research, new methods for the visualization of the unstable bitumen foam, like dynamic X-ray radiography, computed tomography (CT) and high speed camera investigations have been developed. Moreover characterization with empirical methods such as expansion ratio and half-life was determined accurately using ultrasonicmeasurements. This opens new possibilities to characterize the bitumen foam (foaming process) for practical applications. Examination of the foam bitumen stream using high-speed camera revealed that the foam bitumen contains fragmented pieces of bitumen, which resemble more a liquid than a foam. This indicates that the foam is formed afterwards and not, as assumed, within the expansion chamber of the foam generator. In situ thermal imagery of the surface, during the hot foaming process, showed that the temperature distribution depends on the foaming water content and bubble size distribution. Higher water content results in more inhomogeneous temperature distribution as compared to lower water content $(<2$ w-\%). The dynamic X-radiography results indicated that as the foam decays, the bubble size distribution becomes progressively larger with time for $160{ }^{\circ} \mathrm{C}$ bitumen temperature. Furthermore, at the beginning of the foam formation, the majority of the bubbles is small in cross-section size $(0.2-10$ $\mathrm{mm}^{2}$ ). At a later stage the bubbles become polydisperse. Moreover theoretical investigations based on the 3D X-ray CT scan dataset of bubble merging shows that the disjoining pressure increases as the foam film gets thinner with time and finally undergoes rupture. The speed of the bubbles also increases with time when the bubbles are getting closer to each other.
\end{abstract}

Keywords: foam bitumen, bubble merging, X-ray CT scan, experimental investigation 


\section{Introduction}

Different techniques are available to reduce asphalt production and construction temperatures. The use of foam bitumen, as one of them, is attractive due to the low investment and production costs. Foam bitumen is a mixture of air, water and bitumen. It is produced by the injection of small water amounts, typically $1-6 \%$ regarding the bitumen mass, into hot penetration grade bitumen $\left(160-180{ }^{\circ} \mathrm{C}\right)$ in an expansion chamber as shown in Fig. 1(b). The liquid water is transferred into vapor which expands the bitumen to about 5 - 15 times of its original volume [1] forming a foam. This bitumen foam is highly efficient in wetting and coating the surface of fine particles but has difficulties to coat coarse aggregates [2]. As the foam collapses, most of the water is lost as steam, leaving residual bitumen with properties similar to the original bitumen [3].

Foam bitumen allows the production of so called foam asphalt, which is a mixture of mineral aggregates and foam bitumen. Foam asphalt is widely used for cold-in-place recycling (full depth recycling), which is gaining recognition and popularity worldwide as a cost effective method for rehabilitating distressed asphalt pavements [4]. However, the mechanical performance of these foam stabilized pavements is only suitable for sub-base layers but not adequate for base or binder courses, due to insufficient aggregate coating and high air void contents $[5,6]$.

In the traditional hot mix asphalt process the aggregates are heated to $180{ }^{\circ} \mathrm{C}$ and higher. By reducing the temperature to $115{ }^{\circ} \mathrm{C}$, the energy consumption is decreased by about $40 \%$ and $\mathrm{CO}_{2}$ emissions by $31 \%$. Even higher energy savings are feasible if the production temperature can be reduced further. Moreover, asphalt produced at lower temperature does not cool as rapidly, hence allowing longer hauling distances and/or time periods for compacting the material. This generates new opportunities for constructing road pavements in different ways, in particular higher flexibility to perform late/earlyseason paving, thus extending the paving season.

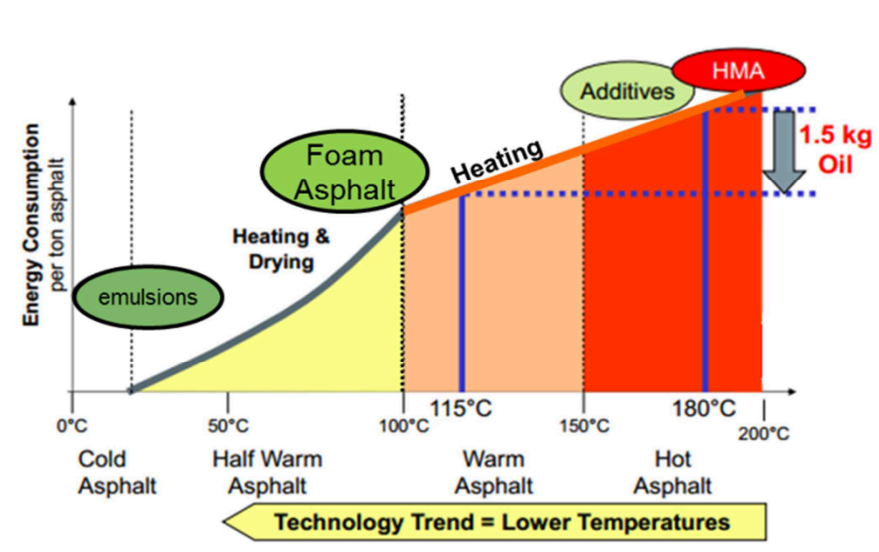

(a)

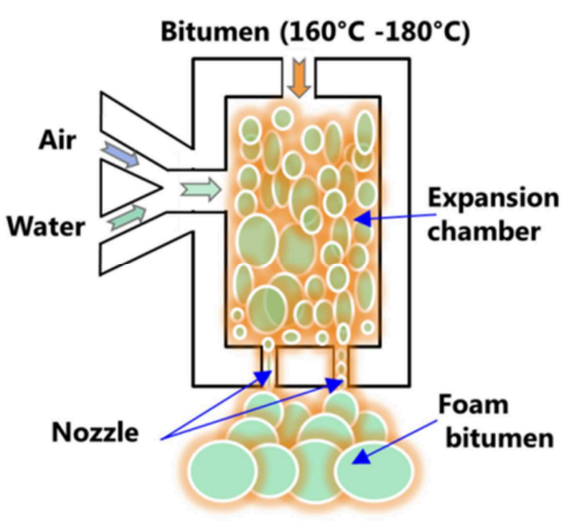

(b)

Figure 1: (a) Scheme of different types of asphalt technology energy consumption[7]; (b) expansion chamber foam bitumen technology. 
Compared to hot mix asphalt (HMA) produced at $150-180^{\circ} \mathrm{C}$, as shown in Fig. 1(a), temperatures for production, transportation, laying and compaction are significantly lower for foam asphalt. Recent developments consisted in the use of heated aggregates to produce warm mix asphalt (WMA 100 $150^{\circ} \mathrm{C}$ ) and half-warm mix asphalt (HWMA, $70-100^{\circ} \mathrm{C}$ ) [8]. In this way coating of the aggregates was considerably improved showing a positive effect on the asphalt properties [6, 9]. However, there are several barriers for wider implementation of foam asphalt such as inadequate coating of coarse aggregates with bitumen at low production temperature [10], improper correlation between laboratory and field results as well as higher air void content resulting in inferior performance of road pavements with foam bitumen $[11,12]$.

The main reason for the missing research on this topic can be explained by the difficult characterization of the foam bitumen. The usual characterization of foam bitumen relies on only two empirical parameters, called expansion ratio and half-life time. However, these two parameters give only the volumetric property of the foam bitumen and provide no information on other foam properties such as foam morphology or bubble size distribution and viscosity. Dynamic processes during foam formation and decay like drainage, coarsening and coalescence remain unclear due to the difficulty to study the unstable and opaque black foam. To the best of our knowledge, no mechanical link between foam viscosity and bitumen viscosity has been established yet.

Although there is a lack of research on bitumen foam, foams have been studied in more detail in other foam application fields (polymer foams, metal foams, soap, coffee, waste water, etc.). Foams are generally classified as dry or wet, depending on the liquid content, which is represented by the liquid volume fraction (Fig. 2). The amount of liquid may range from less than $1 \%$ to above $30 \%$ of the total foam volume. This expression tells only about the foamability (two-phase systems) in general. In dry foams the films forming the interface between bubbles are not spherical in shape, as these bubbles are made of polyhedral cells [13]. Our own preliminary foam bitumen investigations with X-ray radiography showed that foam bitumen consists of disordered spherical bubbles typically found in wet foam[14].

Another classification is based on the porosity of the micro cellular structure. Foams can be classified as open or porous, when voids are completely interconnected, or closed (Fig. 2). In general, both open and closed cells are present in foam. Metal foams commonly have porosity levels less than $70 \%$, whereas ceramic foams show a porosity level between $45 \%$ and $97 \%$. In addition, foams are categorized by how they are formed through chemical or physical processes. For instance, in polymeric materials, thermal decomposition of chemical blowing agents can be used to generate the gas for the expansion of the foam. Mechanical whipping of gases into polymer solution, thus entrapping gases in the matrix, is another way of producing foam. 


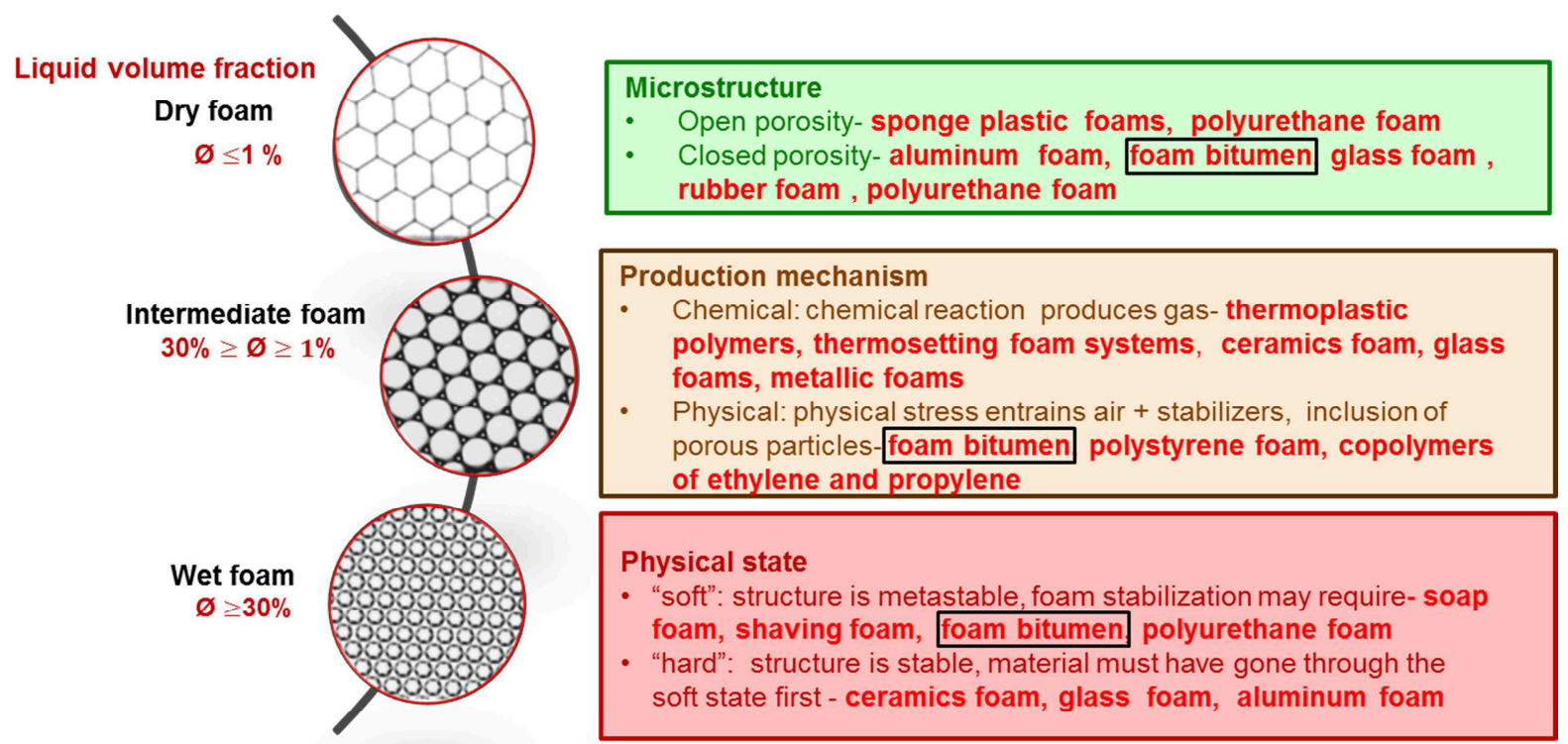

Figure 2: General foam classification [13]

Different mechanisms dominate the stability of a bubble as it moves within the liquid matrix. Initially, bubble growth mainly depends on the pressure balance between the bubble forming gas and the liquid lamella. As the bubble approaches the top surface of the foam, the liquid lamella separating the bubbles gets thinner, leading to dry foam. Then, bubble coalescence and inter bubble gas diffusion starts to govern the foam dynamics. The above phenomena take place in aqueous and non-aqueous foams and were extensively investigated in the past few decades. However, foam bitumen differs from other types of foam in the following ways.

1 Its formation and stability highly depends on the physical process, i.e. the nucleation of bubbles containing water vapor in the hydrophobic hot bitumen. After collapsing, most of the water is lost as steam, leaving residual bitumen with properties similar to the original bitumen[15].

2 The water content and the temperature of the bitumen significantly influences the expansion ratio, which leads, for a wet foam, to a final volumes 5-15 times the starting one [16].

3 Foam bitumen has significant capacity for coating aggregates at lower temperature. Adding water causes coalescence of bubbles that improves the compaction of the asphalt mixture [17].

4 The viscosity of the bitumen has major influence on the bubble size distribution, which depends on the penetration grading of the bitumen and origin of the bitumen [18].

Until now, the characterization of bitumen foam consisted mainly in a visual estimation of the foam expansion by measuring the expansion height of the foam in an open container with a ruler. In this research bitumen foam characterization was experimentally improved by using ultrasonic sensors for accurate monitoring and recording of the foam formation and decay process. In addition, an innovative method for studying the dynamic processes of foam formation and decay was developed using X-ray 
$3^{\text {rd }}$ ISAP APE Symposium Sun City 2015

radiography and computed tomography $(\mathrm{CT})$. This paper focuses on experimental methods and modelling presenting selected data from experiments with different types of bitumen as indicated in Tab. 1 and foamed with different water contents (W.C.).

Table: 1 Penetration and softening properties of bitumen used in the experiments

\begin{tabular}{|c|c|c|}
\hline Type of bitumen & Pen. $(0.1 \mathrm{~mm})$ & $\mathbf{R \& B}\left({ }^{\circ} \mathrm{C}\right)$ \\
\hline A & 81 & 45.3 \\
\hline B & 51 & 51.1 \\
\hline C & 64 & 47.1 \\
\hline
\end{tabular}

\section{Experimental methods}

In order to study the dynamic process of foaming bitumen, a special setup was constructed as shown in Fig. 3 (a). A PMMA (Polymethylmethacrylate) tube with a diameter of $100 \mathrm{~mm}$ was used as foam container for observing the foaming and decaying process through Computed Tomography (CT) scanning. The tube was placed between an X-ray source and detector, providing a field of view (FOV) of $100 \times 100 \times 100 \mathrm{~mm}^{3}$. An extension pipe made of fiber reinforced plastic with an internal diameter of $8 \mathrm{~mm}$ and a length of $2000 \mathrm{~mm}$ was used to connect the foaming unit nozzle and the PMMA tube. Fast X-ray imaging with 14.7 frames per second was used. The rotation speed was $90 \%$ s and enabled acquiring 59 radiographs per $360^{\circ}$ rotation. Since the number of images acquired from the CT scan was considerably less than the minimum number for a normal CT data set(220 images per rotation), a special technique, called simultaneous algebraic reconstruction technique (SART), was applied for reconstructing the tomographic images from the reduced number of radiographs. The CT images had a resolution of $100 \mu \mathrm{m}$ and the theoretical minimum bubble to be detected, had a volume of $8.2 * 10^{-02} \mathrm{~mm}^{3}$.

The tomographic 16-bit images were imported as stack images and each $360^{\circ}$ rotation data set was analyzed separately. The same reconstruction parameters (direction of rotation, filtering technique and center of rotation) were used for each dataset. The specific type of SART algorithm used is the one implemented in the Octopus Reconstruction software suite by Inside Matters[19]. The reconstructed tomographic images were saved in 32-bit format by choosing all range of voxel values using ImageJ to get reasonable brightness and contrast. After reconstruction, each dataset was imported into the Avizo Fire software for 3D image analysis.

The study was conducted with bitumen A at $1 \mathrm{w}-\%$ water content and a bitumen temperature of 160 ${ }^{\circ} \mathrm{C}$. The mass of the bitumen used in the experiment was $100 \mathrm{~g}$. 


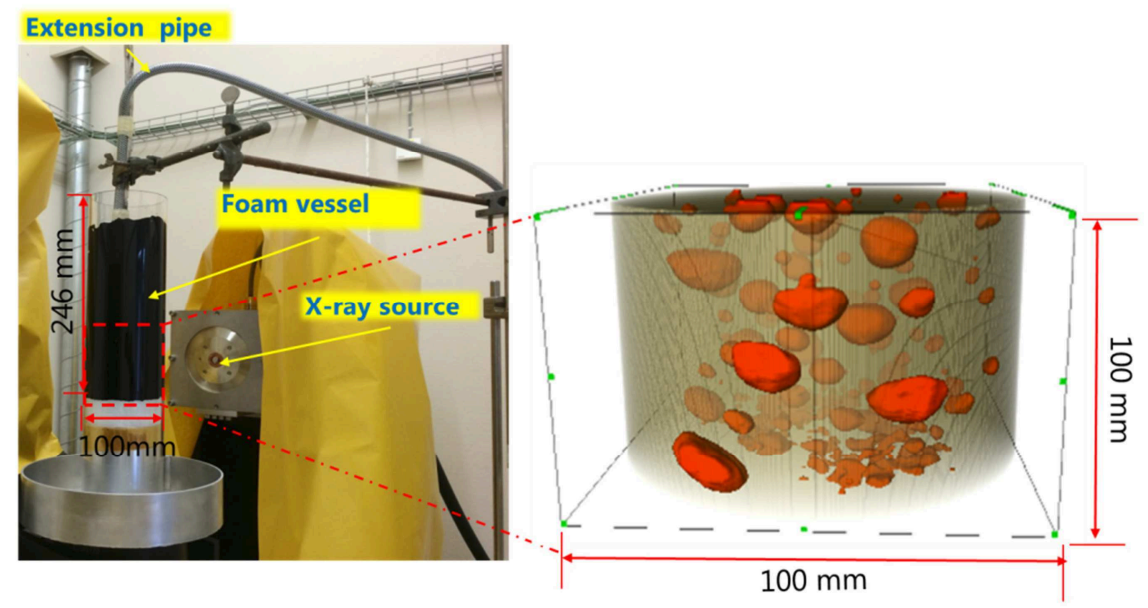

(a)

(b)

Figure 3: (a) X-ray CT scan setup of the foaming experiment; (b) observed bubbles at $~ 40$ s after spraying started

In order to study the bitumen A foam state when leaving the nozzle, a high-speed camera was used for observing the foam bitumen at the four nozzles with $3 \mathrm{~mm}$ diameter openings. As shown in Fig. 4(a) the high speed camera was aligned at $90^{\circ}$ to the foam stream. The setup covered a field of view of 120.0 x $120.0 \mathrm{~mm}$ with a spatial resolution of 2016 x 2016 pixels, corresponding to $\sim 60 \mu \mathrm{m} / \mathrm{pixel}$.

Generally, expansion ratio and half-life are measured by placing a foam ruler next to the side of a container filled with foam bitumen. The half-life is determined with a stopwatch. This measurement is simple and practical. However, since the results can be affected by the operator's skill and experience, repeatability and accuracy are questionable [20]. In this study, measuring foam height of bitumen B over time during foam formation and decay was improved experimentally by using ultrasonic sensor technology (Baumer® standard ultrasonic sensor).

The foam bitumen decays in a highly dynamic and unstable process. Hence, the temperature of the foam can influence the viscosity of the foam and therefore the mixing and coating process. Therefore, the temperature of foam bitumen $\mathrm{C}$ was measured at specific positions by using a series of thermocouples. Simultaneously, the expansion and decay of the foam bitumen was measured with the same ultrasonic sensor as used for A. In the setup shown in Fig. 4(b), three thermocouples were lined on one side of the vessel that contained the foam. Two thermocouples were placed on the bottom and one was used to measure the temperature at the tip of the nozzle. 


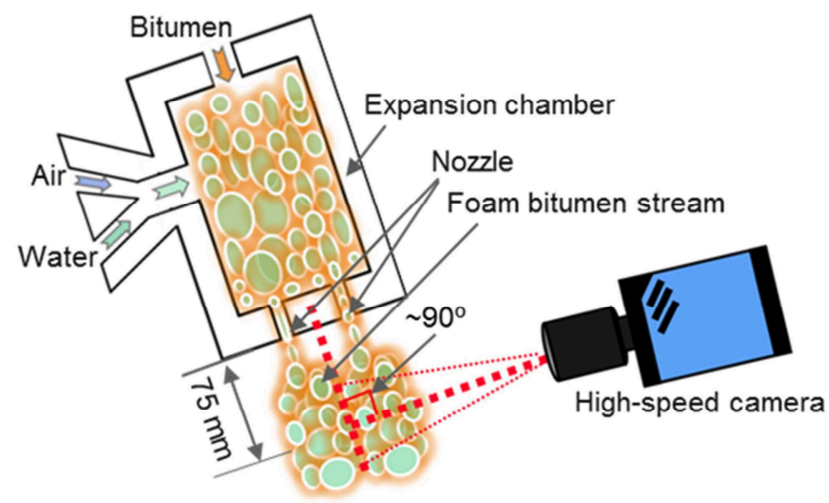

(a)

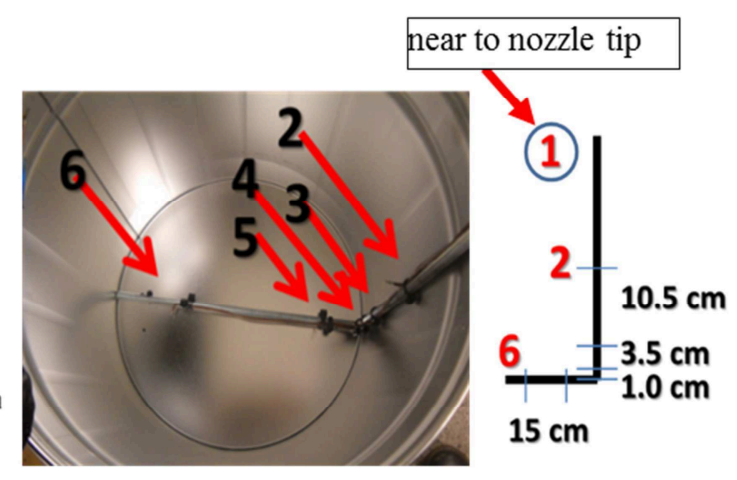

(b)

Figure 4: (a) Schematic of foam bitumen stream observation using high-speed camera; (b) arrangement of thermocouples $1 \ldots 6$ for measuring foam bitumen temperature

\section{Theoretical background}

From general foaming technology it is known that the individual foam bubbles approach each other when they grow. Experimental and theoretical investigations [21-23] on different foaming materials, including polymer and aqueous foams, demonstrate that during this process a flat film can appear between the closest regions of the approaching bubbles, as shown in the Fig. 5(a) and (b). Hydrodynamic interactions as well as buoyancy, electrostatic, van der Waals and steric forces, together with other interactions, can be involved in the formation of the film [24, 25]. According to the extended DeryaginLandau-Verwey-Overbeek (DLVO) theory, the disjoining pressure $\Pi$ responsible for the thinning of a liquid film consists of three types of forces [26, 27]: the attractive Van der Waals interaction $\left(\Pi_{\mathrm{vdw}}\right)$, the repulsive electrostatic force $\left(\Pi_{\mathrm{el}}\right)$, depending on the surface charge properties, and the steric force $\left(\Pi_{\mathrm{st}}\right)$, which depends on the nature and adsorbed species in the presence of a solvent, but significant when the film thickness is below $20 \mathrm{~nm}$ [21, 24]. Other forces such as oscillatory structural forces, hydration and capillary forces occur due to structural ordering at the interface and are active at a separation distance in the order of $4 \mathrm{~nm}$ [24]. Different researchers studied extensively the disjoining pressure $(\Pi)$ in relation to film thickness in order to understand the stability of thin liquid films.

The velocity of the bubbles moving against each other under the action of an external force can be calculated using the Reynolds equation (1) which gives the velocity of the film thinning [24]. Depending on the approaching speed the bubbles behavior can be described in the following terms; stable $(0.01$ $0.04 \mathrm{~mm} / \mathrm{s})$, transiently stable $(\sim 0.04 \mathrm{~mm} / \mathrm{s}-1.2 \mathrm{~mm} / \mathrm{s})$, instant coalescence $(>1.2 \mathrm{~mm} / \mathrm{s}-140$ $\mathrm{mm} / \mathrm{s})[28]$.

$$
V_{\mathrm{Re}}=\frac{P_{L} h^{3}\left(r_{1}^{2}+r_{2}^{2}\right)}{3 \pi \eta r_{1}^{3} r_{2}^{3}}
$$

Where $P_{L}=P c-\Pi ; P_{c}$ is the capillary pressure, $\Pi$ is the disjoining pressure, $r_{1}$ and $r_{2}$ are the radius of bubble, $\eta$ is the dynamic viscosity of the fluid, $h$ is the thickness of the film. 
Fundamental results have been obtained over the years and extensive reviews were made for validating equation (1). For instance, G.E Charles and S.G Mason [29] investigated oil and water bubble film rupture and analyzed experimental data with Reynolds equation (1). Moreover, Vassili V. et al. $[30,31]$ studied the film thinning process in aqueous solution between two air phases in a thin film using Reynolds equation for experiments with a pressure balance where they could control the thinning speed while simulating bubbles approaching each other. Further studies on the film thinning process showed that the equation also works when surfactants are used for stabilizing the liquid foam provided that the assumption holds that the amount of surfactant at the surface remains constant during the thinning process and that there are opposing interfacial fluxes [25]. In addition, other researchers [22, 25, 32] showed experimentally that the film thinning process with and without surfactant and electrolyte is in agreement with Reynolds equation (1).

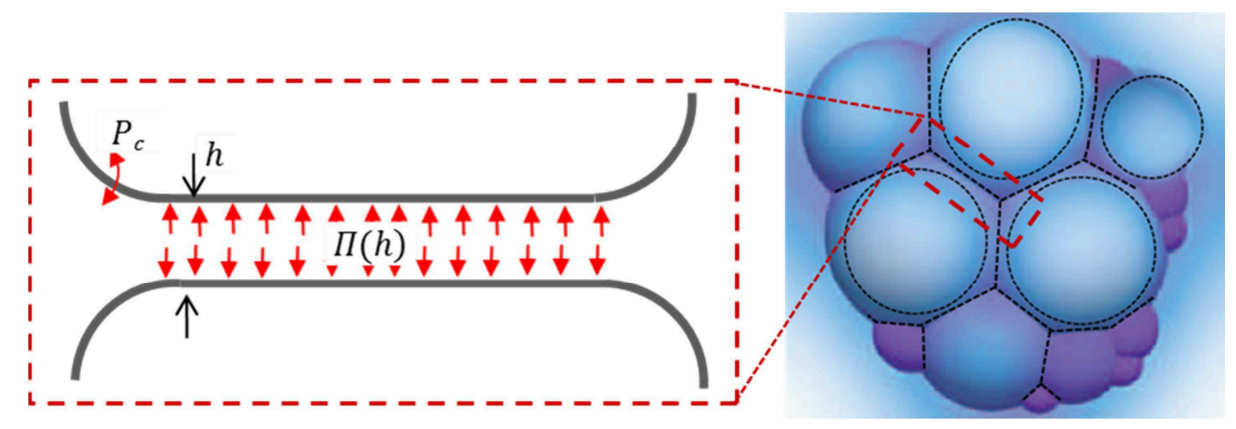

(a)

(b)

Figure 5: (a) Schematic description of a dry foam thin film between bubbles; (b) schematic description of a bubble group.

Foam instability rises from high surface energy associated to the gas-liquid interface, lower viscosity and diffusion of gas from the foam bubbles to the atmosphere. Thermodynamically unstable liquid foams are critical issues in different areas, such as oil recovery, fire extinguishing, food and cosmetics as well as in medicine to produce light-weight structures [33, 34].

Foam collapse can be caused by coarsening or bursting of bubbles on the surface of the foam. Foam coarsening mainly occurs in two ways: by the rupture of the film between two adjacent bubbles or by Ostwald ripening [35]. The rupture of thin films is related to the drainage (redistribution of liquid) and stability of foam films [36], whereas the Ostwald ripening is caused by inter-bubble gas diffusion by which liquid foam comes into thermodynamic equilibrium, where one large bubble is energetically more favorable than two smaller bubbles [37, 38]. On macroscopic scale previous research studies on polymer/gas solutions have shown that rheological parameters such as viscosity and surface tension have a considerable influence on the foaming properties [39, 40]. Similarly the bitumen viscosity (pen. grade) as well as bitumen origin, which can influence the surface tension, has influence on the bubble size distribution $[14,18]$. 


\section{Results and discussion}

Bitumen A was used in this experiment to observe the foam bitumen stream at the outlet nozzle of the foamer. The time indicated in Fig. 6 (a) was measured after the spraying started. The image taken at $1.6 \mathrm{~s}$ reveals that the foam flows in a stream when it comes out of the nozzle. Water droplets were observed in the close-up of the stream during spraying, as depicted in Fig. 6 (b). This shows that the evaporation of the water droplets is not as fast and the foam is formed completely only afterwards and not in the expansion chamber of the foam generator.

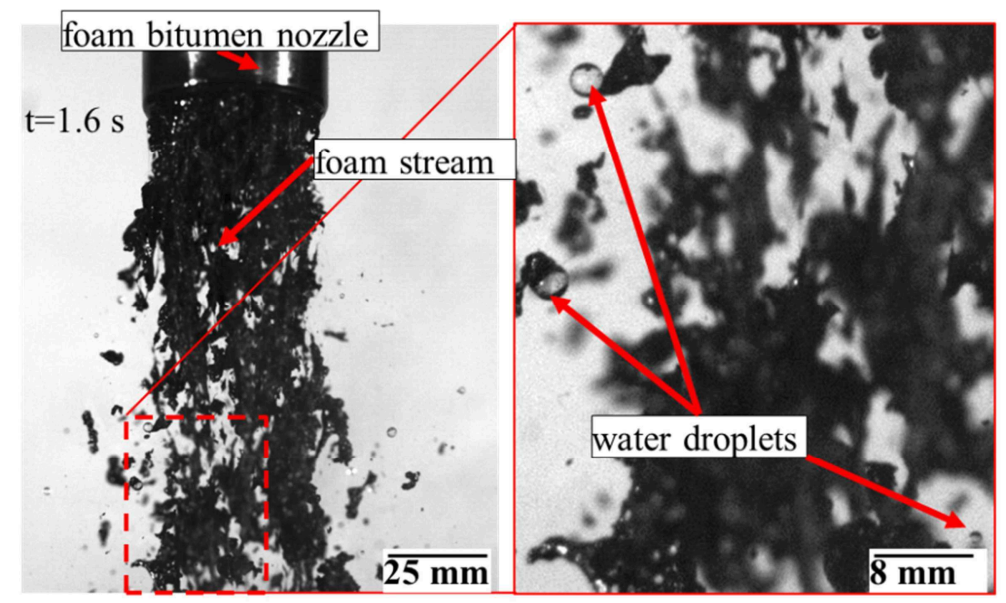

(a)

(b)

Figure 6: Foaming of bitumen A at the nozzle opening: (a) foam bitumen stream details at $4 \mathrm{w}-\%$ water content and $160^{\circ} \mathrm{C}$ bitumen temperature; (b) a close-up of the stream at $1.6 \mathrm{~s}$

In order to study the influence of water content on foam bitumen expansion and decay, foaming experiments were conducted at bitumen temperatures $160^{\circ} \mathrm{C}$ with water contents of $1,2,3,4,5$ and $6.6 \mathrm{w}$ $\%$. The raw data from the ultrasonic sensors are shown in Fig. 7 (a). They were smoothed with a moving average filter method in MATLAB. The expansion and decay curves depicted in Fig. 7 (b) for bitumen $\mathrm{B}$ result from the average of three tests. The results show clearly the influence of water content on foam bitumen decay: as the water content increases, the expansion ratio, which is the ratio of expanded volume in the foamed state to the non-foamed volume of bitumen, increases and the stability of the foam decrease. 


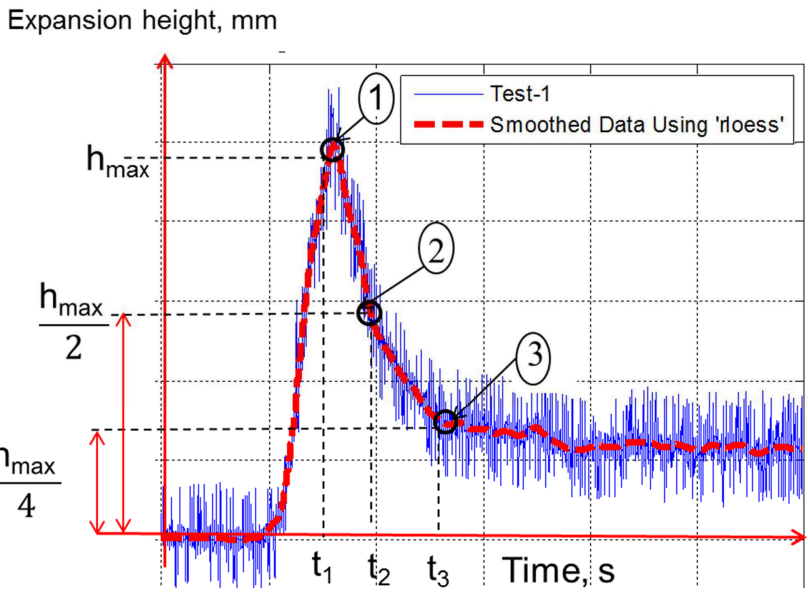

(a)

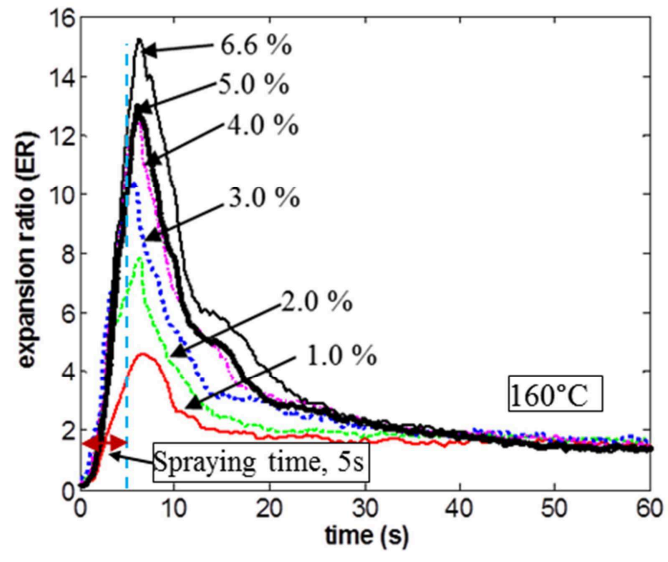

(b)

Figure 7: (a) Foam decay as a function of expansion height and time; (b) influence of water content on foam bitumen expansion and decay for bitumen $\mathrm{B}$.

For determining the foam bitumen temperature during the foaming and decay process, the maximum foam temperature profile was constructed by connecting the maximum temperature envelope of each curve as shown in Fig. 8 (a). The temperature measurement results from the setup shown in Fig. 4 (b) indicate that the foam temperature near the nozzle tip is close to $150{ }^{\circ} \mathrm{C}$ for $2 \mathrm{w}-\%$ W.C. and $146{ }^{\circ} \mathrm{C}$ for 4 w-\% W.C as presented in Fig. 8 (b). These results show clearly the influence of water content on foam bitumen temperature during the decay. The difference in foam temperature curves can be related to uneven distribution of water during the foaming process, which leads to non-uniform temperature distribution in the foam bitumen as shown in Fig. 9 (a). The thermal images in Fig 9 (a) and (b) for the surface temperature of the foam bitumen about $7 \mathrm{~s}$ after start of spraying and after $12 \mathrm{~s}$ suggest the most of the decay is over after a very short period of time. Maximum peak temperature points on the thermal images in Fig. 9 (a) and (b) show bubbles rising to the foam bitumen surface and exploding.

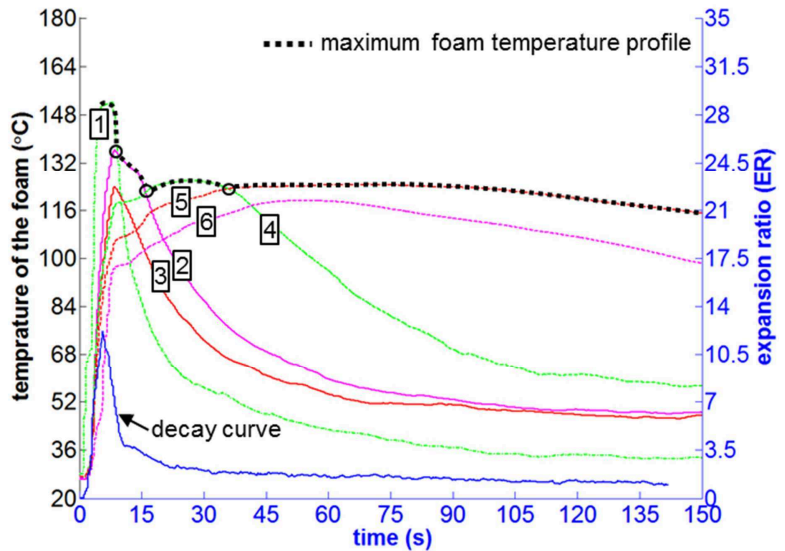

(a)

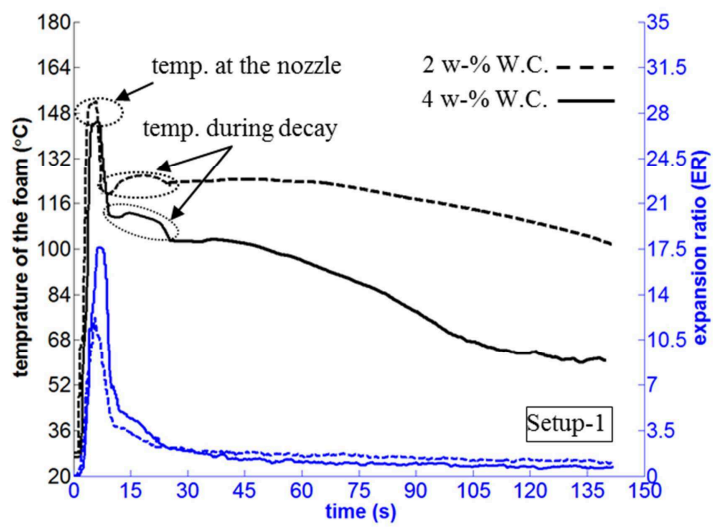

(b)

Figure 8. (a) Foam bitumen temperature and expansion ratio for bitumen $\mathrm{C}$ at different positions 1- 6 and the reconstructed maximum time-temperature profile for $2 \mathrm{w}-\%$ water content; (b) reconstructed temperature data and expansion ratio for $2 \mathrm{w}-\%$ and $4 \mathrm{w}-\%$ water content for bitumen $\mathrm{C}$ 


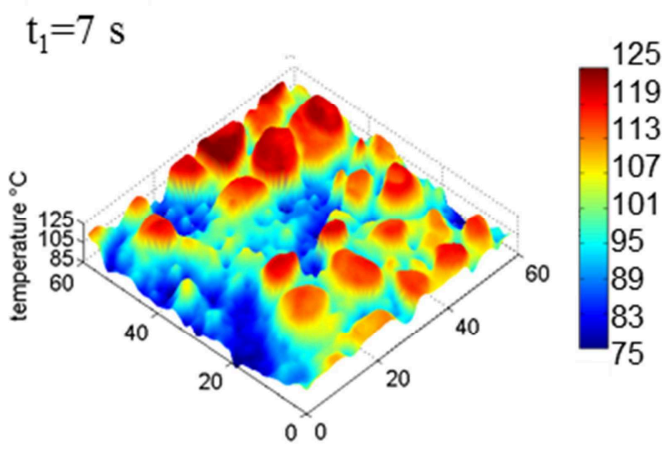

(a)

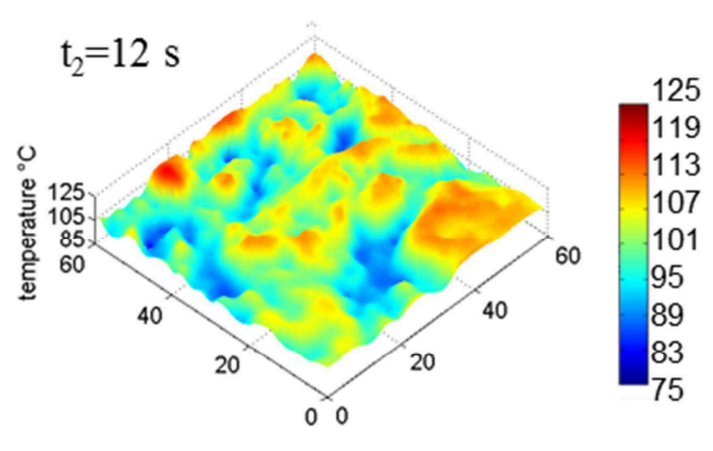

(b)

Figure 9. Surface temperature distribution for bitumen C for $4 \%$ W.C.; (a) at $t_{1}$ and (b) at $t_{2}$ as $\left(t_{1}\right.$ and $t_{2}$ are positions shown in figure 7 a).

Foaming and decay of foam bitumen was also studied by X-ray radiography (2D projection only) as described by Hailesilassie et al. in an earlier paper [18] . The foam analysis section and the representative foam height at the center of the test box are shown in Fig. 10 (a). As an example, Fig. 10 (c) - (e) show the morphology of bubbles at $1 \mathrm{w}-\%$ water content at different heights, i.e. at maximum expansion, half of maximum expansion and one-fourth according to Fig. 7 (a). X-ray radiography experiments are vital to study the distribution and morphology of the foam in $2 \mathrm{D}$ projections, at different times. The cumulative sum of bubble counts shown in Fig. 10 (b) corresponds to the bubble size distribution calculated from the images presented in Fig. 10 (c) - (e). The results show that the count of smaller bubbles $\left(<10 \mathrm{~mm}^{2}\right)$ decreases immensely with decaying foam over time at the beginning of the observation. For bitumen B it was found that the major decaying or destabilizing mechanism was coarsening and bursting of bubbles at the foam bitumen surface. Coarsening is caused by rupture of films between two adjacent bubbles. Because of the polydispersion of foam bitumen, a pressure difference between the inside and outside of the bubbles' surface exists. As a consequence, diffusion of vapor occurs during the decaying process resulting in Ostwald ripening that can lead to foam coarsening [41].

From observing the image sequences, the decay mechanism of foam with bitumen A, with $1 \mathrm{w}-\%$ W.C. and $160{ }^{\circ} \mathrm{C}$ bitumen temperature was found to be mainly caused by merging, drainage and gas loss from bursting bubbles located on the surface of the foam. Drainage of bitumen was not significant. Therefore no separation between the dry foam on the top surface and the wet zone at the bottom of the foam could be seen. 


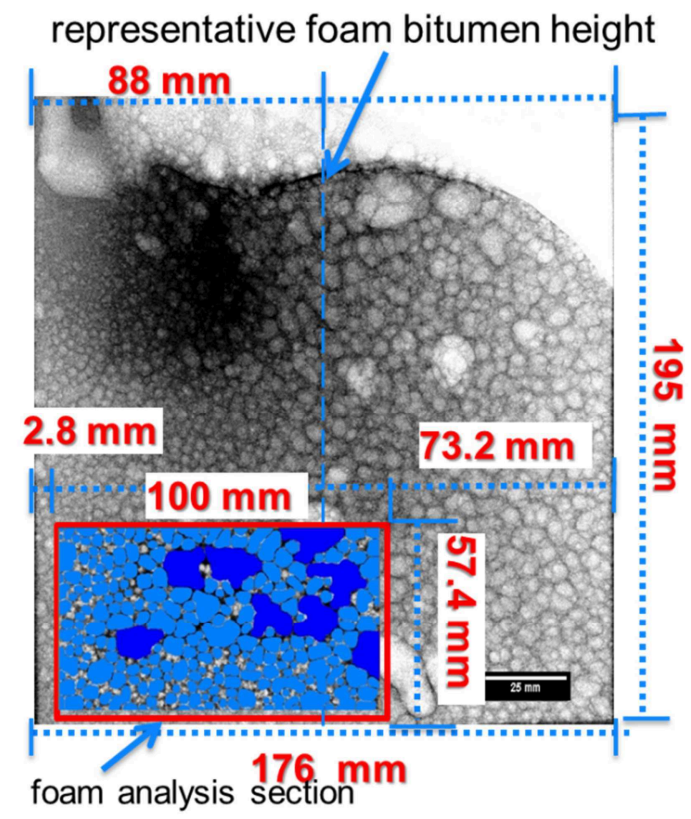

(a)

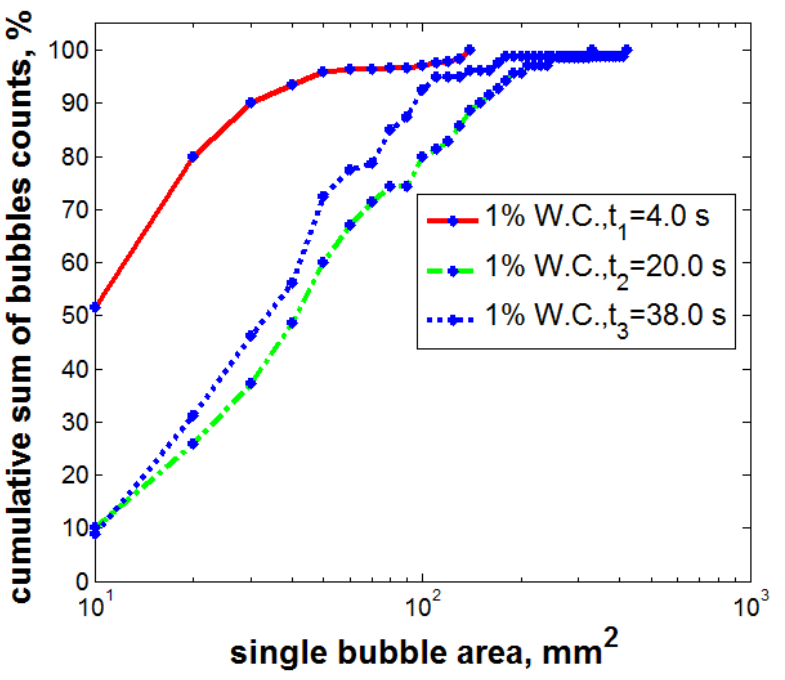

(b)

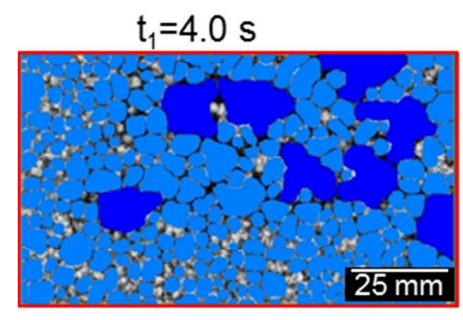

(c)

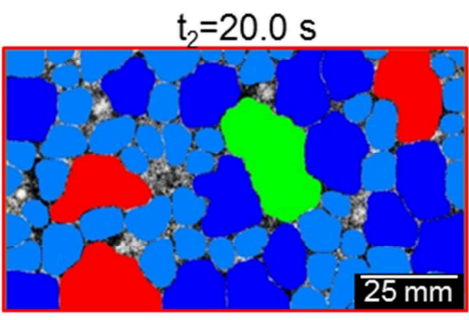

(d)

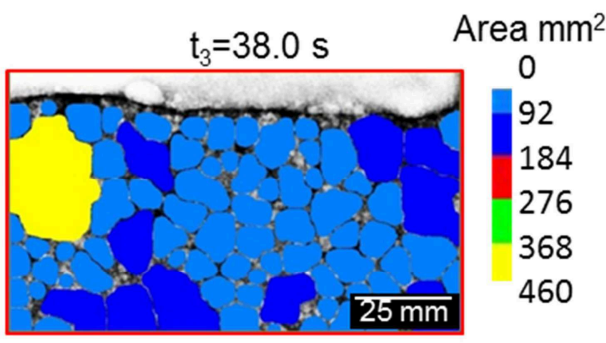

(e)

Figure 10 (a) Foam analysis section by X-ray radiography; (b) cumulative sum of bubbles as a function of single bubble area; (c), (d) and (e) bubble morphology at $h_{\max }, h_{\max / 2}$ and $h_{\max / 4}$ respectively

The dynamic decay process of foam bitumen A was observed in 3D with computed tomography (CT). As seen from Fig. 11 merging of bubbles can be detected during foam decay. Hence, the 3D analysis of individual bubbles appeared the most useful method to obtain quantitative information about the size, shape and arrangement of bubbles. From these experiments, movements of bubbles could be tracked and their velocity of approaching each other could be determined with equation (1). Such information was used to calculate the thinning process between bubbles. As shown in Fig. 12 (a), the trajectory of the bubbles are determined using displacement vector equation (2). The arrows indicate the direction of each bubble displacement.

$$
\Delta F(x, y, z)=\left(\frac{d F}{d x}, \frac{d F}{d y}, \frac{d F}{d z}\right)
$$

Where $F$ is the position of the bubble at $x, y$ and $z$ axis. 


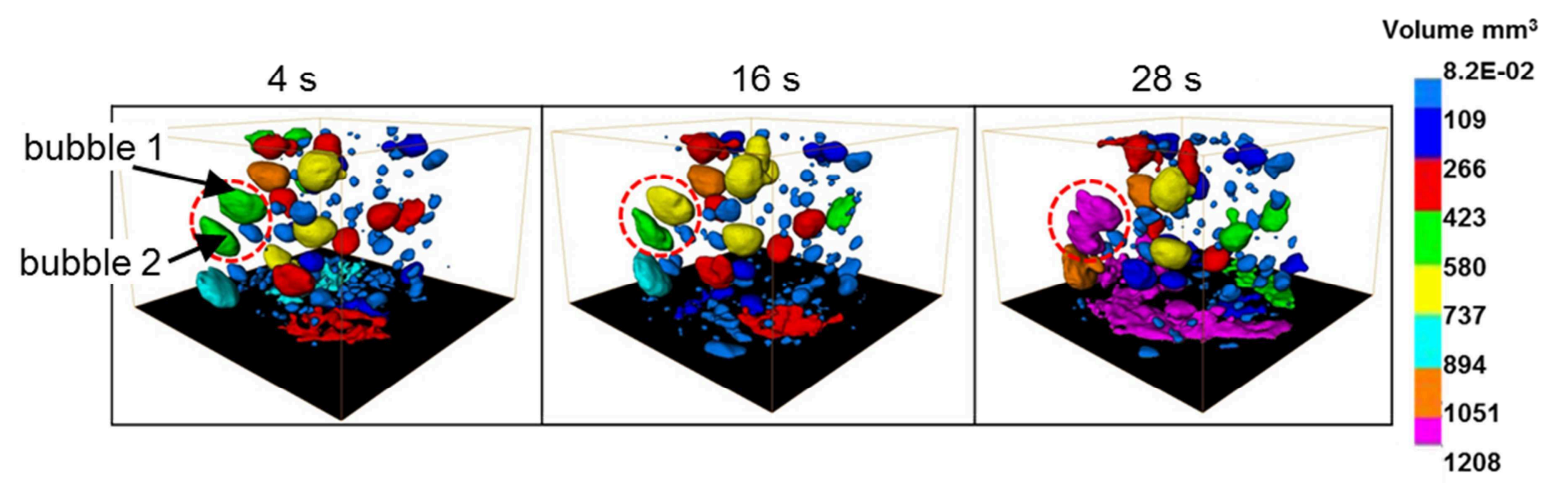

(a)

(b)

(c)

Figure 11: 3D X-ray CT scan of foamed bitumen bubbles at different decay time (every $12 \mathrm{~s}$ ): (a) and (b) bubbles approaching each other; (c) merging of two bubbles due to thinning of the thin film between the bubbles

The velocities of the bubbles were calculated by considering the displacement of foam bubbles over a 4s time interval between each data set step. As shown in Fig. 12 (b) the approaching speed of bubbles is $>1.2 \mathrm{~mm} / \mathrm{s}$ which is leads to instant merging. In order to determine the film thickness at different times, the volumes indicated in Tab. 2 were used to calculate the radius of the bubbles. Spherical bubble shape was assumed for determining the radius. Knowing the distance between centroid of the bubbles and the radius of each bubble at different times (as shown in Tab. 2), it was possible to calculate the bitumen film thickness (i.e. the thickness of the bitumen lamella between the bubbles) as a function of time. The results presented in Tab. 2 correspond to the bubbles merging shown in Fig. 11 (c).

Moreover, the disjoining pressure (П) was calculated from (3) by rearranging equation (1).

$$
P_{c}-\Pi=V_{\operatorname{Re}} \frac{3 \pi \eta r_{1}^{3} r_{2}^{3}}{h^{3}\left(r_{1}^{2}+r_{2}^{2}\right)}
$$

When the bubbles approach, a flat film can appear between the closest regions of the bubbles as shown in Fig. 5 (a) and Fig. 11(b). As a result, the capillary pressure $P_{c}$ can be close to zero or neglected. The dynamic viscosity of the bitumen was 2.2 Pa.s (at $110{ }^{\circ} \mathrm{C}$ ) and radius of the bubbles, $\mathrm{r}_{1}$ and $\mathrm{r}_{2}$, are indicated in Tab. 2. The velocity of film thinning $\left(V_{R e}\right)$ is determined by taking ratio $\Delta \mathrm{h} / \Delta \mathrm{t}$. The disjoining pressure (П) calculated with equation (3), shows increase of disjoining pressure when the bubbles approach each other. Finally, this leads to rupture of the thin film and as a result the bubbles merge. 


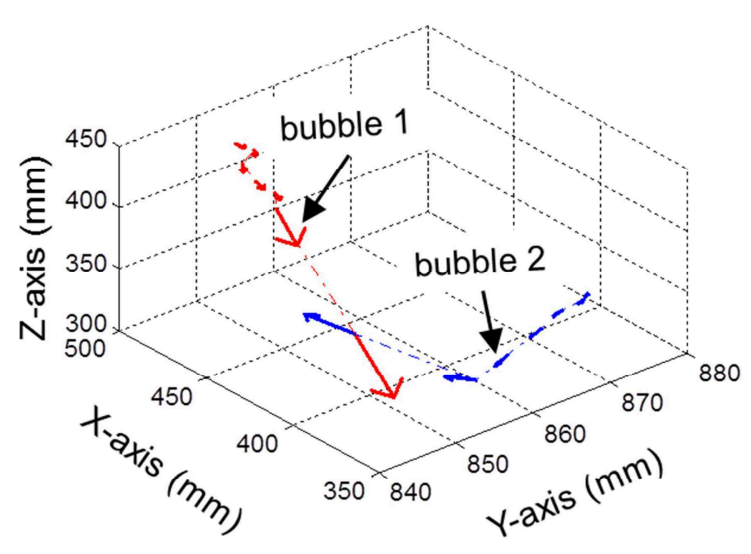

(a)

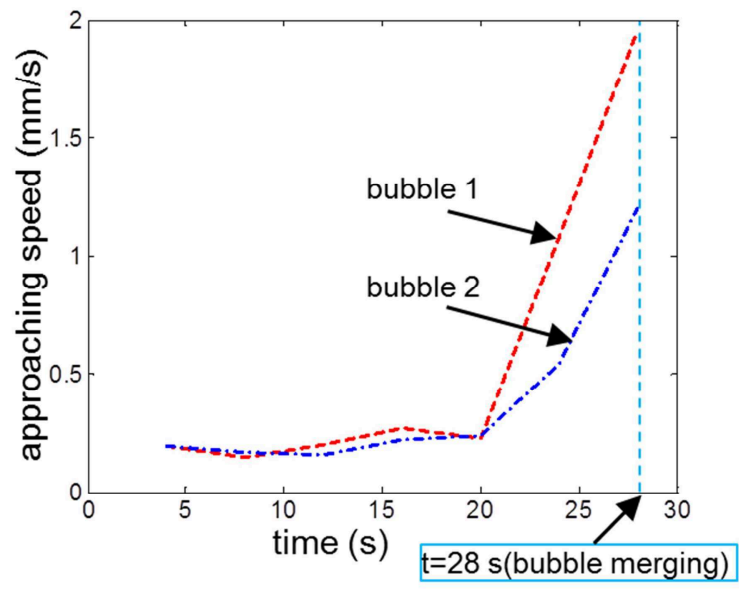

(b)

Figure 12: (a) Bubble trajectory movement path from CT scan dataset; the arrows indicate the displacement vector; (b) speed of bubbles as a function of time

Table 2: geometrical data from X-ray CT scan, film thickness and disjoining pressure at different time

\begin{tabular}{|c|c|c|c|c|c|c|c|}
\hline \multicolumn{3}{|c|}{ Bubble 1} & \multicolumn{2}{|c|}{ Bubble 2} & \multirow{2}{*}{$\begin{array}{l}\text { Distance between } \\
\text { centroid of Bubbles } \\
(\mathrm{mm})\end{array}$} & \multirow{2}{*}{$\begin{array}{c}\text { Film thickness, } \\
\mathrm{h}(\mathrm{mm})\end{array}$} & \multirow{2}{*}{$\begin{array}{c}\text { Pressure, } \\
\Pi(\mathrm{Pa})\end{array}$} \\
\hline $\begin{array}{l}\text { Time } \\
\text { (s) }\end{array}$ & $\begin{array}{c}\text { volume } \\
\left(\mathrm{mm}^{3}\right)\end{array}$ & $\begin{array}{l}\text { Radius, } \\
\mathrm{r}_{1}(\mathrm{~mm})\end{array}$ & $\begin{array}{c}\text { volume } \\
\left(\mathrm{mm}^{3}\right)\end{array}$ & $\begin{array}{l}\text { Radius, } \\
\mathrm{r}_{2}(\mathrm{~mm})\end{array}$ & & & \\
\hline 4 & 834.8 & 5.84 & 822.7 & 5.81 & 15.05 & 3.40 & 24.36 \\
\hline 8 & 876.2 & 5.94 & 803.5 & 5.77 & 14.79 & 3.09 & 53.47 \\
\hline 12 & 891.9 & 5.97 & 788.4 & 5.73 & 14.27 & 2.57 & 85.82 \\
\hline 16 & 867.4 & 5.92 & 803.9 & 5.77 & 13.78 & 2.10 & 248.07 \\
\hline 20 & 886.2 & 5.96 & 821.3 & 5.81 & 13.10 & 1.34 & 568.76 \\
\hline 24 & 937.3 & 6.07 & 824.2 & 5.82 & 12.53 & 0.89 & 3634.14 \\
\hline 28 & 1743.8 & 7.47 & 1743.8 & 7.47 & - & 0 & - \\
\hline
\end{tabular}

\section{Conclusion}

This research demonstrated that new research techniques and theoretical calculation can help to understand and improve existing bitumen foaming technology. From the high speed camera image it was discovered that, at the outlet of the nozzle, the bitumen foam has not yet been completely formed. The "foam bitumen" stream showed fragmented pieces of irregular size which resembled rather a liquid state than a foam. This indicates that the expansion of the bubbles in the expansion chamber is not as fast as may be expected. Hence, the foam is formed afterwards and not as assumed in the expansion chamber of the foam generator.

The ultrasonic sensor measurement used for measuring the foam bitumen expansion height is repeatable and gives accurate results. This technique also helps to determine the half-life of the foam bitumen correctly. 
The thermal images indicate that the distribution of maximum and minimum peak temperature depends on the bubble size distribution. In addition, the amount of water used for foaming the bitumen influences the bubble size distribution. The foam bitumen bubbles are larger when using 4 w-\% W.C. compared to $1 \mathrm{w}-\%$ W.C. as seen in the X-ray radiography measurements.

The new testing method developed using X-ray radiography (2D representation) for observing the formation and decay of foam bitumen bubbles is a useful technique to make progress in understanding and characterizing the mechanism of foaming bitumen. Foam bitumen decay was observed as a dynamic coarsening process of foam bitumen bubbles. The coarsening takes place in a fraction of seconds creating impacts on adjacent bubbles and eventually leading to sudden merging of bubbles. In all experiments it was observed that there is a redistribution of bubble size during the decay process. During foam decay, the bubble size distribution became progressively larger for $160{ }^{\circ} \mathrm{C}$ bitumen temperature.

Considerable progress in understanding the principle of the different foaming processes and the phenomena that take place in the foam films was achieved by investigating free standing films. The disjoining pressure increased as the foam film gets thinner with time and finally undergoes rupture. The speed of the bubbles also increases with time when the bubbles are getting closer to each other.

Formation and decay of foam bitumen with and without stabilizer is a complex mechanism which certainly needs further attention and research efforts. It was found that the foam bitumen formation and decaying mechanism elaborated in this paper are helpful to understand the influence of water and temperature on the foam bitumen from the experimental point of view. Understanding the decaying mechanism helps to identify and suggest stabilizing mechanisms.

\section{Reference}

[1] Koenders B. G., Stoker D. A., Bowen C., de Groot P., Larsen O., Hardy D., and Wilms K. P., "Innovative Process In Asphalt Production And Application To Obtain Lower Operating Temperatures", presented at the 2nd Eurasphalt \& Eurobitume Congress Barcelona 2000, 2000.

[2] Jenkins K. J., "Mix Design Consideration for Cold and Half-Warm Bituminous Mixes with Emphasis on Foam Bituemn ", Doctor of Philosphy, Departement of Civil Engineering university of Stellenbosch, 2000.

[3] Namutebi M., "Some Aspects of Foamed Bitumen Technology", Licentiate Thesis, Division of Highway and Railway Engineering, Royal Institute of Technology, Stockholm, 2011.

[4] Romanoschi S. A., Hossain M., Heitzman M., and Gisi A. J., "Foamed Asphalt Stabilized Reclaimed Asphalt Pavement: A Promising Technology for Mid-Western Roads ", in Proceedings of the 2003 Mid-Continent Transportation Research Symposium, Ames, Iowa, August 2003.

[5] Yin F., Cucalon L. G., Martin A. E., Arambula E., Chowdhury\$ A., and Park E. S., "Laboratory conditioning protocols for warm-mix asphalt ", presented at the AAPT 2013 Annual Meeting and Technical Sessions, 2013.

[6] Jenkins K. J., " Half-warm foamed bitumen treatment, a new process", presented at the 7th Conference on Asphalt Pavements for Southern Africa CAPSA 1999. 
[7] Bieder A., Probst S., Biedermann A., and Demarmels A., "Foaming of Bitumen - a key Process for Diffrent low Temperature Asphalts ", presented at the 5th Eurasphalt \& Eurobitume Congress, Istanbul, 2012.

[8] van de Ven M. F., Sluer B. W., Jenkins K. J., and van den Beemt M. A., "New developments with half-warm foamed bitumen asphalt mixtures for sustainable and durable pavement solutions", Road Materials and Pavement Design, vol. 13, pp. 713-730, 2012.

[9] Chowdhury A. and Button J. W., "A Review of Warm Mix Asphalt", Texas Transportation Institute, 2008.

[10] Hailesilassie B. W., Hugener M., and Partl M. N., "Influence of foaming water content on foam asphalt mixtures", Construction and Building Materials, vol. 85, pp. 65-77, 2015.

[11] Van De ven M. F. C., Jenkins K. J., Voskuilen J. L. M., and Van Den Beemt R., " Development of (half-) warm foamed bitumen mixes: state of the art", International Journal of Pavement Engineering, vol. 8, pp. 163-175, 2007.

[12] Rubio M. C., Martínez G., Baena L., and Fernando Moreno, " Warm mix asphalt: an overview", Journal of Cleaner Production 24, pp. 76-84, 2012.

[13] Stevenson P., Foam Engineering. United Kingdom: A John Wiley \& Sons, Ltd., 2012.

[14] Hailesilassie B. W., Schuetz P., Jerjen I., Bieder A., Hugener M., and Partl M. N., "Evolution of Bubble Size Distribution during Foam Bitumen Formation and Decay", presented at the ISAP 2014 Conference, 2014.

[15] Jenkins K. J., "Mix design considerations for cold and half-warm bituminous mixes with emphasis on foamed bitumen ", Doctor of philosophy department of civil engineering, University of Stellenbosch south Africa, 2000.

[16] O.R. Larsen, Ø. Moen, C. Robertus, and Koenders B. G., "Wam foam aspalt production at lower operating temperatures as an enviromental friendly alternative to HMA", presented at the 3rd Eurasphalt \& Eurobitume Congress Vienna, 2004.

[17] Joe W. Button and Arif Chowdhury, "A Review of Warm Mix Asphalt ", Texas Transportation Institute, 2008.

[18] Hailesilassie B., Schuetz P., Jerjen I., Hugener M., and Partl M. N., "Dynamic X-ray radiography for the determination of foamed bitumen bubble area distribution", Journal of Materials Science, vol. 50, pp. 79-92, 2015.

[19] http://insidematters.eu/octopus. (2015).

[20] He G.-p. and Wong W.-g., "Decay properties of the foamed bitumens", Construction and Building Materials, vol. 20, pp. 866-877, 12// 2006.

[21] Exerowa D., Kolarov T., Pigov I., Levecke B., and Tadros T., "Interaction Forces in Thin Liquid Films Stabilized by Hydrophobically Modified Inulin Polymeric Surfactant. 1. Foam Films", Langmuir, vol. 22, pp. 5013-5017, 2006.

[22] Manica R., Klaseboer E., and Chan D. Y. C., "Dynamic interactions between drops-a critical assessment", Soft Matter, vol. 4, pp. 1613-1616, 2008.

[23] Karraker K. A. and Radke C. J., "Disjoining pressures, zeta potentials and surface tensions of aqueous non-ionic surfactant/electrolyte solutions: theory and comparison to experiment", Advances in Colloid and Interface Science, vol. 96, pp. 231-264, 2002.

[24] Handbook of Surface and Colloid Chemistry, Third Edition: CRC press, 2008.

[25] Stockle S., Blecua P., Mohwald H., and Krastev R., "Dynamics of thinning of foam films stabilized by n-dodecyl-beta-maltoside", Langmuir, vol. 26, pp. 4865-72, 2010.

[26] Vance B., "Forces and structure in thin liquid soap films", Journal of Physics Condensed Matter, vol. 11, p. R215, 1999.

[27] Cosima S. and Regine von K., "Disjoining pressure in thin liquid foam and emulsion filmsnew concepts and perspectives", Journal of Physics Condensed Matter, vol. 15, p. R1197, 2003.

[28] Del Castillo L. A., Ohnishi S., and Horn R. G., "Inhibition of bubble coalescence: Effects of salt concentration and speed of approach", Journal of Colloid And Interface Science, vol. 356, pp. 316-324, 4/1/ 2011.

[29] Charles G. E. and Mason S. G., "The coalescence of liquid drops with flat liquid/liquid interfaces", Journal of Colloid Science, vol. 15, pp. 236-267, 6// 1960.

[30] Yaminsky V. V., Ohnishi S., Vogler E. A., and Horn R. G., "Stability of Aqueous Films between Bubbles. Part 1. The Effect of Speed on Bubble Coalescence in Purified Water and 
Simple Electrolyte Solutions", Langmuir, vol. 26, pp. 8061-8074, 2010.

[31] Yaminsky V. V., Ohnishi S., Vogler E. A., and Horn R. G., "Stability of Aqueous Films between Bubbles. Part 2. Effects of Trace Impurities and Evaporation", Langmuir, vol. 26, pp. 8075-8080, 2010.

[32] Wang L. and Yoon R.-H., "Role of hydrophobic force in the thinning of foam films containing a nonionic surfactant", Colloids and Surfaces A: Physicochemical and Engineering Aspects, vol. 282-283, pp. 84-91, 2006.

[33] Larry L. Hench and Julia M. Polak, "Third-Generation Biomedical Materials", Science, vol. 295, pp. 1014-1017 2002.

[34] Gonzenbach U. T., Studart A. R., Tervoort E., and Gauckler L. J., "Stabilization of foams with inorganic colloidal particles", Langmuir, vol. 22, pp. 10983-10988, 2006.

[35] Lemlich R., "Prediction of Changes in Bubble Size Distribution due to Interbubble Gas Diffusion in Foam.", Ind. Eng. Chem. Fundam, vol. 17, pp. 89-93, 1978.

[36] Stevenson P., Foam Engineering Fundamentals and Applications, 2012.

[37] Lambertm J., Cantat I., Delannay R., Mokso R., Cloetens P., Glazier J., and Graner F., "Experimental Growth law for Bubbles in a Moderately "wet" 3D Liquid Foam", Phys. Rev. Lett., vol. 99, 2007.

[38] Rami-shojaei S., Vachier C., and Schmitt C., "Automatic analysis of 2D foam sequences: Application to the characterization of aqueous proteins foams stability", Image and Vision Computing, vol. 27, 2009.

[39] R Höhler and S Cohen-Addad, "Topical Review: Rheology of liquid foam", Journal of Physics Condensed Matter, vol. 17, pp. R1041-R1069, 2005.

[40] Simon Cox, Denis Weaire, and James A. Glazier, "The rheology of two-dimensional foams", Rheol Acta, vol. 43, pp. 442-448, 2004.

[41] Denis W. and Stefan H., The Physics of Foams. oxford: clarendon press 1999. 